D) Check for updates

Cite this: Chem. Commun., 2021, 57,6141

Received 27th March 2021 , Accepted 14th May 2021

DOI: $10.1039 / \mathrm{d} 1 \mathrm{cc} 01650 \mathrm{~d}$

rsc.li/chemcomm

\title{
Creating porosity in a trianglimine macrocycle by heterochiral pairing $\dagger$
}

\author{
Donglin He, (D) Rob Clowes, Marc A. Little, (D) * Ming Liu (D) * and \\ Andrew I. Cooper (D)*
}

\begin{abstract}
Macrocycles are usually non-porous or barely porous in the solid-state because of their small intrinsic cavity sizes and tendency to close-pack. Here, we use a heterochiral pairing strategy to introduce porosity in a trianglimine macrocycle, by co-crystallising two macrocycles with opposing chiralities. The stable racemic trianglimine crystal contains an interconnected pore network that has a Brunauer-Emmett-Teller (BET) surface area of $355 \mathrm{~m}^{2} \mathrm{~g}^{-1}$.
\end{abstract}

Porous materials are widely useful in applications such as gas storage, molecular separations, and sensing of gases or vapours. ${ }^{1-4}$ Synthetic control over pore structure and topology has been achieved for extended framework materials such as zeolites, ${ }^{5}$ metal-organic frameworks (MOFs) ${ }^{6}$ and covalent organic frameworks (COFs). ${ }^{7}$ There is also a growing interest in porous molecular solids; one such example is porous organic cages (POCs), ${ }^{8-10}$ where solid state function can be intrinsic to the molecular building units. Porous molecular crystals have some potential advantages compared with their extended framework cousins, such as their improved processibility. ${ }^{11,12}$ In general, however, it is also more challenging to design structure and hence function for porous molecular solids because their crystal packing is often dictated by the sum of a variety of relatively weak and often non-directional intermolecular forces.

Macrocycles, whose solution-phase host-guest chemistry has been studied extensively, ${ }^{13}$ have been explored recently for a range of molecular separations using the macrocycles in the solid, crystalline state. For example, Janiak and co-workers reported a trianglamine macrocycle crystal with 1-D channels that absorbed ethanol. ${ }^{14}$ We reported a trianglimine macrocycle crystal that can separate ethyl acetate from its azeotropic mixture with ethanol. ${ }^{15}$ Eddaoudi and co-workers reported a

Materials Innovation Factory and Chemistry Department, University of Liverpool, Liverpool, L7 3NY, UK. E-mail: aicooper@liverpool.ac.uk

$\dagger$ Electronic supplementary information (ESI) available. CCDC 2073632-2073637. For ESI and crystallographic data in CIF or other electronic format see DOI: 10.1039/d1cc01650d triangleamine-based supramolecular organic framework that showed permanent porosity and high affinity for $\mathrm{CO}_{2} \cdot{ }^{16} \mathrm{We}$ also reported formally non-porous pillar[ $n]$ arenes that selectively adsorbed styrene from ethylbenzene ${ }^{17}$ and para-xylene from its structural isomers. ${ }^{18}$ However, a challenge to the practical use of macrocycles as adsorbents for separations is their limited adsorption capacities and (often) slow adsorption kinetics. One strategy to solve these kinetic and capacity problems is to increase the porosity in the molecular system. For example, 3-D POCs, with larger cavities can exhibit Brunauer-Emmett-Teller surface areas $\left(\mathrm{SA}_{\mathrm{BET}}\right)$ as high as $3758 \mathrm{~m}^{2} \mathrm{~g}^{-1} \cdot{ }^{19,20}$ It is more challenging to introduce significant porosity into macrocycles. This is because macrocycles have lower dimensional intrinsic porosity; they are also prone to close packing, minimizing any void space in the solid state. ${ }^{21,22}$ Hence, unlike POCs with 3-D intrinsic connected pores in the solid state, macrocycle solids rarely have interconnected pore networks that facilitate the rapid diffusion of guest molecules. To date, examples of macrocycles that have been reported with a specific surface area higher than $100 \mathrm{~m}^{2} \mathrm{~g}^{-1}$ are still rare. ${ }^{18,23}$ There is also potential benefit in accessing porous macrocycle crystals with porosity levels that are high enough to permit good diffusion kinetics while retaining the small and size/ shape-specific cavity of the macrocycle.

One approach to create porosity in macrocycle structures is to introduce "extrinsic" pores that connect to the small intrinsic macrocycle pores. ${ }^{24}$ Modular co-crystallization strategies have proven to be effective here. ${ }^{22}$ For example, the modular assembly of POCs of opposing chiralities has made it possible to control the size and shape of pores in POC crystals. ${ }^{25,26}$ The same approach also allowed 'gating POCs' to be combined with a second POC to exclude a competitive guest and to achieve high guest selectivity. ${ }^{27}$ However, to our knowledge, this method has not been applied to control the solid state porosity of intrinsically porous molecules, other than POCs.

Isotrianglimine [3+3] macrocycles, formed by reacting isophthalaldehyde with aliphatic diamines, were first reported by Gawronski and co-workers in $2000 .{ }^{28}$ Subsequently, a series of 
a
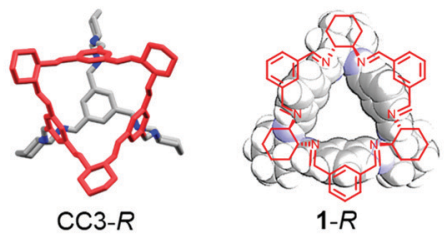

b

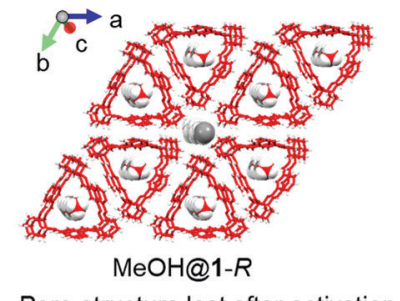

Pore structure lost after activation

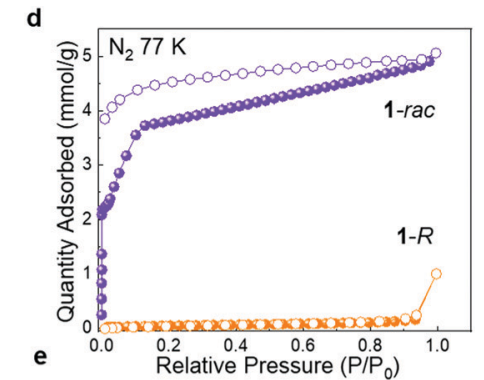

c
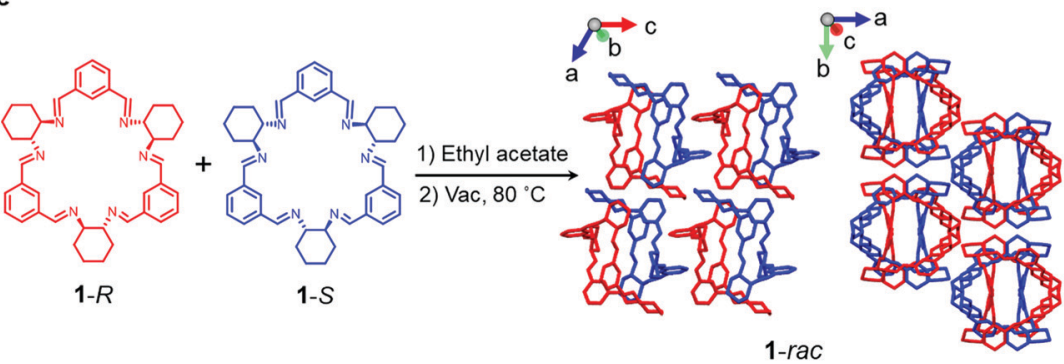

Pore structure retained after activation

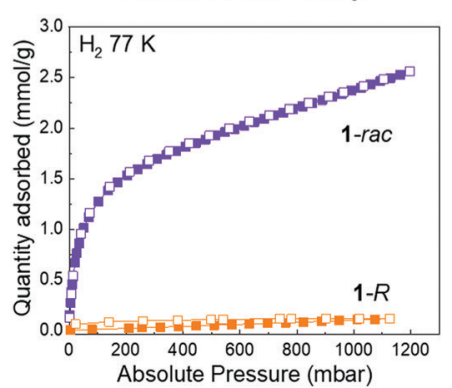

Fig. 1 (a) Single crystal structures of the chiral POC, CC3- $R$, which contains a structural fragment equivalent to 1-R (left), and 1- $R$ (red) displayed in space filling mode (right). (b) Crystal packing in $\mathrm{MeOH} @ 1-R, \mathrm{MeOH}$ guest displayed in space filling mode. (c) Single crystal structure of 1-rac. 1- $R$ (red) and 1-S (blue); $\mathrm{H}$ atoms are omitted for clarity. (d) $\mathrm{N}_{2}$ and (e) $\mathrm{H}_{2}$ sorption isotherms at $77 \mathrm{~K}$ for activated 1- $R$ (orange) and 1-rac (purple). Solid symbols: adsorption; hollow symbols: desorption.

chiral isotrianglimines were developed, formed by the condensation of trans-1,2-cyclohexanediamine with substituted isophthalaldehydes were reported, ${ }^{29,30}$ including 1- $R$ (Fig. 1a) that is synthesised by reacting trans-1,2-cyclohexanediamine with isophthalaldehyde. The structure of $1-R$ is reminiscent of the window motif in the chiral POC CC3-R (Fig. 1a), which we showed to direct the crystal packing of POCs by generating energetically favourable heterochiral window-to-window packing motifs in racemic crystals. ${ }^{26}$

Here, we obtained solvated single crystals of 1- $R$ from methanol (MeOH@1- $R$, Fig. 1b) that revealed the 1- $R$ molecules were stacked in an eclipsed fashion along the crystallographic $c$-axis. This motif gave rise to pillars of $1-R$ that contained $\mathrm{MeOH}$ in their intrinsic cavities. In this structure, the neighbouring 1-R pillars are linked by intermolecular hydrogen bonding interaction via the methanol solvent molecules. It was therefore unsurprising that the packing of $1-R$ changed when the $\mathrm{MeOH}$ molecules were removed from the MeOH@1- $R$ crystals (Fig. S7, ESI $\dagger$ ) to afford a polycrystalline 1- $R$ sample that was confirmed to be non-porous to both $\mathrm{N}_{2}$ and $\mathrm{H}_{2}$ (Fig. 1d and e).

Solvated racemic co-crystals of 1 (EA@1-rac) were obtained by recrystallising an equimolar ratio of 1- $R$ and 1-S from ethyl acetate (EA, Fig. 1c). These crystals were found to remain suitable for single crystal analysis after activation under a dynamic vacuum at $80{ }^{\circ} \mathrm{C}$. In the activated structure, 1-rac, the racemic window-to-window packing between macrocycles is stabilised by $\pi-\pi$ stacking and $\mathrm{C}-\mathrm{H} \cdots \pi$ interactions, and this was retained after removing the EA solvent (Fig. 1c and Fig. S8, S12, ESI $\dagger$ ). The assembly of neighbouring 1-rac heterochiral pairings along the $c$-axis generates interconnected pores in the crystal structure that occupy $11.6 \%$ of the unit cell, as calculated using Platon with a probe radii of $1.2 \AA$ (Fig. 1c), with the largest free sphere $\left(D_{\mathrm{f}}\right)$ of $2.31 \AA$ calculated by Zeo++. ${ }^{31}$ It should be noted that the 1-R structure crystallised from EA was also barely porous to $\mathrm{N}_{2}$ and $\mathrm{H}_{2}$ after activation (Fig. S7, S17a and b, ESI $\dagger$ ), highlighting the importance of the racemic pairing motif for generating porosity in 1-rac.

To evaluate the gas sorption properties of 1- $R$ and 1-rac, we used the probe gases, $\mathrm{N}_{2}, \mathrm{H}_{2}$, and $\mathrm{CO}_{2}$. The $\mathrm{N}_{2}$ isotherms (Fig. 1d) show that 1-rac is porous to $\mathrm{N}_{2}$, with an apparent $\mathrm{SA}_{\mathrm{BET}}$ of $355 \mathrm{~m}^{2} \mathrm{~g}^{-1}$, and that it undergoes low-pressure adsorption step at 0.66 mbar. By contrast, $1-R$ is essentially non-porous to $\mathrm{N}_{2}$ and has a much lower apparent $\mathrm{SA}_{\mathrm{BET}}$ of $4 \mathrm{~m}^{2} \mathrm{~g}^{-1} \cdot{ }^{32}$ 1-rac also has a much higher $\mathrm{H}_{2}$ uptake at 1 bar and

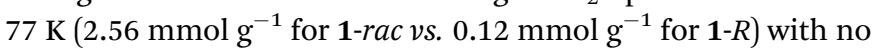
hysteresis found for either sample (Fig. 1e). The gas sorption isotherms confirm that we successfully created porosity in macrocycle 1 by using the heterochiral pairing strategy to stabilise a porous crystal packing.

For materials containing very small or disconnected pores, which $\mathrm{N}_{2}$ molecules cannot access at cryogenic temperatures, $\mathrm{CO}_{2}$ isotherms are often used to probe porosity. ${ }^{33}$ For $1-R$, the rapid onset of $\mathrm{CO}_{2}$ adsorption isotherm at very low pressure at $195 \mathrm{~K}$ indicates the presence of ultra-fine pores that cannot be accessed by $\mathrm{N}_{2}$ molecules at $77 \mathrm{~K}$ (Fig. S17a, ESI $\dagger$ ). ${ }^{34} \mathrm{CO}_{2}$ uptake for 1-rac shows a typical type-I isotherm with no hysteresis loop at $195 \mathrm{~K}$; at 1 bar, 1-rac absorbed significantly more $\mathrm{CO}_{2}$ than 1- $R$ (3.80 mmol vs. $2.17 \mathrm{mmol}$ ) (Fig. 2a). This result is consistent with the $\mathrm{N}_{2}$ isotherms and confirms that substantial additional porosity has been created in 1-rac. The high pressure $\mathrm{CO}_{2}$ adsorption isotherm of 1-rac at $273 \mathrm{~K}$ shows two successive 'plateaus' (Fig. 2b), which corresponds to $\mathrm{CO}_{2}$ accessing the different pores in the flexible 1-rac structure as it expands, and 
a

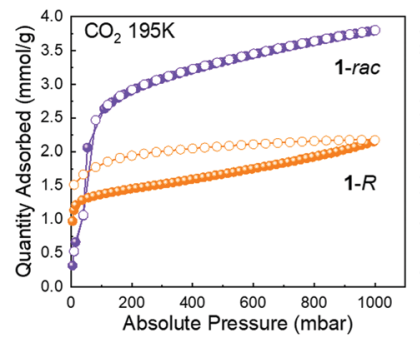

b

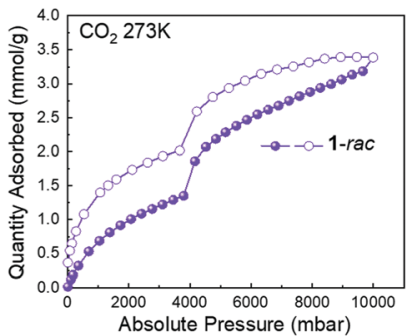

Fig. $2 \mathrm{CO}_{2}$ isotherms of 1-rac (violet) and 1- $R$ (orange): (a) $\mathrm{CO}_{2}$ isotherms at $195 \mathrm{~K}$ form $0-1$ bar. (b) $\mathrm{CO}_{2}$ isotherms at $273 \mathrm{~K}$ from 0 to 10 bar. Adsorption isotherms as closed symbols; desorption isotherms as open symbols.

similar behaviour has been observed in other porous solids. ${ }^{35}$ At $273 \mathrm{~K}, 283 \mathrm{~K}$, and $298 \mathrm{~K}, 1-R$ adsorbs more $\mathrm{CO}_{2}$ than $1-r a c$ in the low relative pressure range (0-1 bar). To understand this initially counterintuitive phenomenon, the isosteric heats of adsorption $\left(Q_{\mathrm{st}}\right)$ were calculated from the $\mathrm{CO}_{2}$ isotherms (273-298 K, 0-1 bar) for 1-rac and 1-R. As shown in Fig. S17d (ESI $\dagger)$, the calculated isosteric heats of adsorption $\left(Q_{\mathrm{st}}\right)$ for $1-R$ and 1-rac were less than $30 \mathrm{~kJ} \mathrm{~mol}^{-1}$, excluding chemisorption by either adsorbent. ${ }^{36}$ The $Q_{\text {st }}$ for $\mathrm{CO}_{2}$ on 1- $R$ remained constant over a larger adsorbate loading range $\left(0-1 \mathrm{mmol} \mathrm{g}^{-1}\right)$ indicating an energetically homogeneous surface. ${ }^{37}$ 1-rac had a linear decrease in $Q_{\text {st }}$ with $\mathrm{CO}_{2}$ loading over the range of $0-0.5 \mathrm{mmol} \mathrm{g}^{-1}$, indicating that 1-rac is more energetically heterogeneous for the adsorption of $\mathrm{CO}_{2} \cdot{ }^{37}$ The higher $\mathrm{CO}_{2}$ uptake of 1- $R$ at lower pressures can be attributed to ultra-fine pores in 1- $R$ that can adsorb $\mathrm{CO}_{2}$ as a monolayer. By contrast, the larger interconnected pores of 1-rac lead to the multilayer adsorption of $\mathrm{CO}_{2}$ but with lower uptakes at lower pressures.

The separation of xylene isomers is challenging because they have similar molecular structures and physical properties. Recently, molecular materials such as cucurbit[7]uril ${ }^{38}$ and the polymorphic azobenzene cage ${ }^{39}$ have been studied as adsorbents for the separation of xylene isomers. The largest included sphere along the free sphere path $\left(D_{\mathrm{if}}\right)$ calculated by Zeo++ $+^{31,40}$ in activated 1-rac is $4.26 \AA$, which is close to the molecular size of para-xylene $(p \mathrm{X})(4.2 \times 6.8 \AA)$ (Table S1, ESI $\dagger)$. This close size match suggested that 1-rac might be a good host for $p \mathrm{X}$ over its structural isomer, meta-xylene $(m \mathrm{X}$, Table $\mathrm{S} 1$, ESI $\dagger$ ). We initially crystallised $1-R$ and $1-S$ from $p \mathrm{X}$ and found the resulting inclusion complex, 1pX@1-rac (Fig. 3a). The crystal structure of $1 p \mathrm{X} @ 1$-rac revealed that one $p \mathrm{X}$ molecule crystallised in the centre of the cavity created between $1-R$ and 1- $S$ molecules packed in a window-to-window arrangement. A second $p \mathrm{X}$ molecule in the structure was located in an extrinsic void created between four 1 molecules. Compared with the guest-free structure of 1-rac, the inclusion of $p \mathrm{X}$ does not significantly change the packing of $\mathbf{1}$ (Fig. 3a), as confirmed by the crystal packing overlay shown in Fig. 3b. However, 1-rac does expand by around $8 \%$ to accommodate $1 \mathrm{~mol} \mathrm{~mol}^{-1}$ of $p \mathrm{X}$ in its structure. By contrast, the inclusion complex, 3mX@ 2(1-rac), has a different packing mode compared to activated 1-rac (Fig. S9a, S14, and S16, ESI $\dagger$ ). These results suggest that a

a

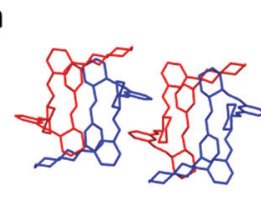

1-rac

b
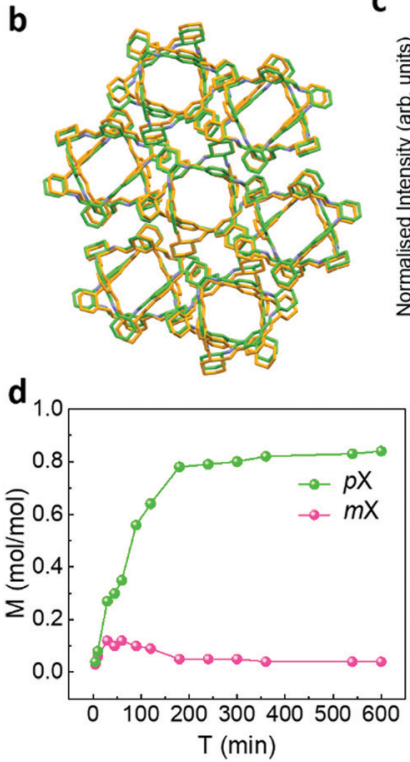

C
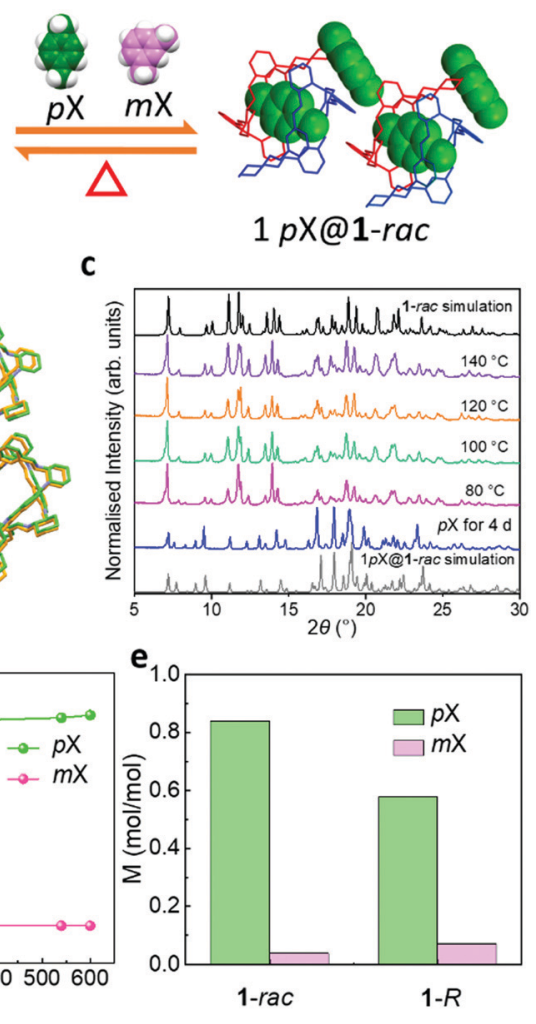

Fig. 3 (a) Reversible capture of $p X$ from 1:1 $p X-m X$ vapour mixture illustrated by single-crystal structures for 1-rac (left) and 1pX@1-rac (right). 1- $R$ and $1-S$ are coloured red and blue, respectively; $\mathrm{H}$ atoms are omitted for clarity. (b) Crystal packing overlay for $1 p X @ 1$-rac (green) and activated 1-rac (yellow). (c) PXRD patterns of 1-rac after being exposed to $p$ X vapour and then dried under vacuum at elevated temperatures. (d) Timedependent 1-rac solid-vapour sorption plot for $p X$ and $m X$ equimolar vapour mixture. (e) The capacity of $1-R$ and $1-r a c$, determined using a $1: 1$ $p X-m X$ vapour mixture.

racemic mixture of $\mathbf{1}$ can form inclusion complexes with both $p \mathrm{X}$ and $m \mathrm{X}$ isomers, but that the crystal packing in guest-free 1-rac is more closely matched for $p \mathrm{X}$ inclusion. When we activated 1pX@1-rac and 3mX@2(1-rac) they transformed into the 1-rac structure at $80^{\circ} \mathrm{C}$ and $140{ }^{\circ} \mathrm{C}$, respectively (Fig. 3c and Fig. S9a, ESI $\dagger$ ), indicating that 1-rac is the energetically favourable phase. However, the lower activation temperature for 1pX@1-rac indicates that $p \mathrm{X}$ desorbs more easily from 1pX@1-rac (Fig. S6, ESI $\dagger$ ), which is likely due to the more interconnected 1-D porosity in 1pX@1-rac (Fig. 3b) and the narrower dimensions of $p \mathrm{X}$.

To determine if 1-rac could separate $p \mathrm{X}$ from $m \mathrm{X}$, we performed time-dependent solid-vapour sorption experiments using the vapours generated from a physical mixture of the two xylene isomers. As shown in Fig. 3d, porous 1-rac captures $p \mathrm{X}$ selectively from a $1: 1$ (vol:vol) mixture of $p \mathrm{X}$ and $m \mathrm{X}$. The maximum uptake of $p \mathrm{X}$ with 1-rac was $0.83 \mathrm{~mol} \mathrm{~mol}^{-1}$ after $10 \mathrm{~h}$, which is close to the ideal ratio of 1 in $1 p \mathrm{X} @ 1-$ rac. The capacity of 1-rac for $p \mathrm{X}$ in $1: 1 p \mathrm{X}-m \mathrm{X}$ vapour mixture is about 1.5 times higher than for $1-R$, which we attribute to the increased porosity in 1-rac (Fig. 3e). A larger difference, however, is in the adsorption kinetics. For example, a formally 
non-porous pillar[6]arene macrocycle that adsorbs $p \mathrm{X}$ over $m \mathrm{X}$ with similar selectivity was found to reach saturation after 20 hours. $^{18}$ 1-rac performs much better than this, reaching saturation after only 3 hours under the same conditions, with a selectivity coefficient of $K_{p \mathrm{X}: m \mathrm{x}}=15.7 .^{41}$ The greatly improved adsorption kinetics of 1-rac compared to the pillar[6]arene system is a direct result of its increased porosity, which results from the chiral pairing strategy.

In conclusion, we have introduced porosity into a trianglimine macrocycle system by using a heterochiral pairing strategy. Porosity was created by co-crystallising two macrocycles with the opposing chiralities such that they pack in a windowto-window arrangement to connect the intrinsic macrocycle voids. This generates an interconnected pore network with an apparent $\mathrm{SA}_{\mathrm{BET}}$ of $355 \mathrm{~m}^{2} \mathrm{~g}^{-1}$. This is the highest reported surface area for the trianglimine macrocycle, ${ }^{16,23}$ which are usually barely porous in the solid state. Because of its increased porosity, the 1-rac co-crystal has greatly improved adsorption kinetics and shows the potential to separate xylene isomers, exhibiting much higher selectivity toward $p \mathrm{X}$, by a factor of 15.7 vs. $m \mathrm{X}$, outperforming related macrocyclic systems for the same separation. ${ }^{18}$ As well as introducing porosity, the heterochiral pairing strategy could also enrich the functionality of these macrocycle systems by enabling hybrid mixing of macrocycles with different functions that would otherwise not co-crystallise, as demonstrated with POCs for quantum sieving applications. $^{42}$

The authors acknowledge the EPSRC (EP/N004884/1) and the Leverhulme Trust via the Leverhulme Research Centre for Functional Materials Design for funding. D. H. thanks the Oversea Study Program of Guangzhou Elite Project provided by Guangzhou City, China for financial support.

\section{Conflicts of interest}

There are no conflicts to declare.

\section{Notes and references}

1 C. Sapsanis, H. Omran, V. Chernikova, O. Shekhah, Y. Belmabkhout, U. Buttner, M. Eddaoudi and K. N. Salama, Sensors, 2015, 15, 18153-18166.

2 M. G. Rabbani and H. M. El-Kaderi, Chem. Mater., 2012, 24, 1511-1517.

3 J.-R. Li, R. J. Kuppler and H.-C. Zhou, Chem. Soc. Rev., 2009, 38, 1477-1504.

4 M. Brutschy, M. W. Schneider, M. Mastalerz and S. R. Waldvogel, Adv. Mater., 2012, 24, 6049-6052.

5 V. M. Georgieva, E. L. Bruce, M. C. Verbraeken, A. R. Scott, W. J. Casteel, S. Brandani and P. A. Wright, J. Am. Chem. Soc., 2019, 141, 12744-12759.

6 S. Ma, X.-S. Wang, D. Yuan and H.-C. Zhou, Angew. Chem., Int. Ed., 2008, 47, 4130-4133.

7 R.-Q. Wang, X.-B. Wei and Y.-Q. Feng, Chem. - Eur. J., 2018, 24, 10979-10983.

8 T. Tozawa, J. T. Jones, S. I. Swamy, S. Jiang, D. J. Adams, S. Shakespeare, R. Clowes, D. Bradshaw, T. Hasell, S. Y. Chong, C. Tang, S. Thompson, J. Parker, A. Trewin, J. Bacsa, A. M. Slawin, A. Steiner and A. I. Cooper, Nat. Mater., 2009, 8, 973-978.
9 T. Mitra, K. E. Jelfs, M. Schmidtmann, A. Ahmed, S. Y. Chong, D. J. Adams and A. I. Cooper, Nat. Chem., 2013, 5, 276-281.

10 A. G. Slater and A. I. Cooper, Science, 2015, 348, aaa8075.

11 J. R. Holst, A. Trewin and A. I. Cooper, Nat. Chem., 2010, 2, 915-920.

12 A. I. Cooper, ACS Cent. Sci., 2017, 3, 544-553.

13 F. Vögtle and E. Weber, Host Guest Complex Chemistry Macrocycles: Synthesis, Structures, Applications, Springer Science \& Business Media, 2012.

14 A. Janiak, M. Bardziński, J. Gawroński and U. Rychlewska, Cryst. Growth Des., 2016, 16, 2779-2788.

15 D. He, C. Zhao, L. Chen, M. Little, S. Chong, R. Clowes, K. McKie, M. Roper, G. Day, M. Liu and A. Cooper, Chem. - Eur. J., 2021, DOI: $10.1002 /$ chem.202101510.

16 A. Chaix, G. Mouchaham, A. Shkurenko, P. Hoang, B. Moosa, P. M. Bhatt, K. Adil, K. N. Salama, M. Eddaoudi and N. M. Khashab, J. Am. Chem. Soc., 2018, 140, 14571-14575.

17 K. Jie, M. Liu, Y. Zhou, M. A. Little, S. Bonakala, S. Y. Chong, A. Stephenson, L. Chen, F. Huang and A. I. Cooper, J. Am. Chem. Soc., 2017, 139, 2908-2911.

18 K. Jie, M. Liu, Y. Zhou, M. A. Little, A. Pulido, S. Y. Chong, A. Stephenson, A. R. Hughes, F. Sakakibara, T. Ogoshi, F. Blanc, G. M. Day, F. Huang and A. I. Cooper, J. Am. Chem. Soc., 2018, 140, 6921-6930.

19 M. Mastalerz, Acc. Chem. Res., 2018, 51, 2411-2422.

20 G. Zhang, O. Presly, F. White, I. M. Oppel and M. Mastalerz, Angew. Chem., Int. Ed., 2014, 53, 1516-1520.

21 N. B. McKeown, J. Mater. Chem., 2010, 20, 10588-10597.

22 M. A. Little and A. I. Cooper, Adv. Funct. Mater., 2020, 30, 1909842.

23 E. Sanna, E. C. Escudero-Adán, A. Bauzá, P. Ballester, A. Frontera, C. Rotger and A. Costa, Chem. Sci., 2015, 6, 5466-5472.

24 M. J. Bojdys, M. E. Briggs, J. T. A. Jones, D. J. Adams, S. Y. Chong, M. Schmidtmann and A. I. Cooper, J. Am. Chem. Soc., 2011, 133, 16566-16571.

25 J. T. A. Jones, T. Hasell, X. Wu, J. Bacsa, K. E. Jelfs, M. Schmidtmann, S. Y. Chong, D. J. Adams, A. Trewin, F. Schiffman, F. Cora, B. Slater, A. Steiner, G. M. Day and A. I. Cooper, Nature, 2011, 474, 367-371.

26 T. Hasell, S. Y. Chong, K. E. Jelfs, D. J. Adams and A. I. Cooper, J. Am. Chem. Soc., 2012, 134, 588-598.

27 T. Hasell and A. I. Cooper, Nat. Rev. Mater., 2016, 1, 16053.

28 J. Gawroński, H. Kołbon, M. Kwit and A. Katrusiak, J. Org. Chem., 2000, 65, 5768-5773.

29 N. Kuhnert, G. M. Rossignolo and A. Lopez-Periago, Org. Biomol. Chem., 2003, 1, 1157-1170.

30 A. Troć, J. Gajewy, W. Danikiewicz and M. Kwit, Chem. - Eur. J., 2016, 22, 13258-13264.

31 T. F. Willems, C. H. Rycroft, M. Kazi, J. C. Meza and M. Haranczyk, Microporous Mesoporous Mater., 2012, 149, 134-141.

32 M. Thommes, K. Kaneko, A. V. Neimark, J. P. Olivier, F. RodriguezReinoso, J. Rouquerol and K. S. Sing, Pure Appl. Chem., 2015, 87, 1051-1069.

33 P. I. Ravikovitch, A. Vishnyakov, R. Russo and A. V. Neimark, Langmuir, 2000, 16, 2311-2320.

34 B. D. Chandler, D. T. Cramb and G. K. Shimizu, J. Am. Chem. Soc., 2006, 128, 10403-10412.

35 P. Lama and L. J. Barbour, J. Am. Chem. Soc., 2018, 140, 2145-2150.

36 K.-J. Chen, D. G. Madden, T. Pham, K. A. Forrest, A. Kumar, Q.-Y. Yang, W. Xue, B. Space, J. J. Perry Iv, J.-P. Zhang, X.-M. Chen and M. J. Zaworotko, Angew. Chem., Int. Ed., 2016, 55, 10268-10272. 37 S. Sircar and D. V. Cao, Chem. Eng. Technol., 2002, 25, 945-948.

38 G. Zhang, A.-H. Emwas, U. F. Shahul Hameed, S. T. Arold, P. Yang, A. Chen, J.-F. Xiang and N. M. Khashab, Chem, 2020, 6, 1082-1096.

39 B. Moosa, L. O. Alimi, A. Shkurenko, A. Fakim, P. M. Bhatt, G. Zhang, M. Eddaoudi and N. M. Khashab, Angew. Chem., Int. Ed., 2020, 59, 21367-21371.

40 M. D. Foster, I. Rivin, M. M. J. Treacy and O. Delgado Friedrichs, Microporous Mesoporous Mater., 2006, 90, 32-38.

41 A. M. Pivovar, K. T. Holman and M. D. Ward, Chem. Mater., 2001, 13, 3018-3031.

42 M. Liu, L. Zhang, M. A. Little, V. Kapil, M. Ceriotti, S. Yang, L. Ding, D. L. Holden, R. Balderas-Xicohténcatl, D. He, R. Clowes, S. Y. Chong, G. Schütz, L. Chen, M. Hirscher and A. I. Cooper, Science, 2019, 366, 613-620. 